\title{
An accurate formula for the period of a simple pendulum oscillating beyond the small angle regime
}

\author{
F. M. S. Lima* \\ Instituto de Física, Universidade de Brasilia, \\ P. O. Box 04455, 70919-970, Brasília-DF, Brazil \\ P. Arun \\ Department of Physics and Electronics, SGTB Khalsa College, \\ University of Nova Delhi, Delhi 110 00\%, India
}

\begin{abstract}
A simple approximate formula is derived for the dependence of the period of a simple pendulum on the amplitude. The approximate is more accurate than other simple formulas. Good agreement with experimental data is verified.
\end{abstract}




\section{INTRODUCTION}

The periodic motion exhibited by a simple pendulum is harmonic only for small angle oscillations. $\stackrel{1}{-}$ Beyond this limit, the equation of motion is nonlinear. Although an integral formula exists for the period of the nonlinear pendulum, it is usually not discussed in introductory physics classes because it is not possible to evaluate the integral exactly ${ }^{2}$ For this reason almost all introductory physics textbooks and lab manuals discuss only small angle oscillations for which the approximation $\sin \theta \approx \theta$ is valid. The linearized equation has a simple exact solution, whose derivation can be easily understood by first-year students. 1 This linearization has bothered us since our own undergraduate days because the amplitude needs to be less than $7^{\circ}$ if an error less than $0.1 \%$ (the typical experimental error obtained with a stopwatch) is desired. Measurements in undergraduate labs rarely have such small amplitudes, $\underline{\underline{3}}$ and interested students sometimes ask for a relation that can describe the increase of the period observed for large amplitudes. 4

The restriction to small angle oscillations hinders the understanding of real-world behavior because the pendulum isochronism observed in the small angle regime vanishes for increasing amplitudes. This restriction is also unnecessary because millisecond precision in measurements of the period is easily obtained with current technology ${ }^{5.6 .7 .8}$ For instance, an experimental error of the order of $0.1 \%$ or less is typically obtained with a one meterlong pendulum, and thus accurate experimental studies of the dependence of the period on amplitude are possible even in introductory physics labs. ${ }^{7.8}$

In this paper we derive a simple and accurate formula for the period of a pendulum oscillating beyond the small angle regime. The deviation from the exact results are of the same order of the experimental error.

\section{APPROXIMATION}

An ideal simple pendulum consists of a particle of mass $m$ suspended by a massless rigid rod of length $L$ that is fixed at the upper end such that the particle moves in a vertical circle. This simple mechanical system oscillates with a symmetric restoring force (in the absence of dissipative forces) due to gravity, as illustrated in Fig. 1. Its equation of motion 
is given by $\underline{2}^{2}$

$$
\frac{d^{2} \theta}{d t^{2}}+\frac{g}{L} \sin \theta=0
$$

where $\theta$ is the angular displacement in radians $(\theta=0$ at the equilibrium position) and $g$ is the local acceleration of gravity. For any given initial condition, the exact solution can only be obtained numerically (with arbitrary accuracy). For small angle oscillations, the approximation $\sin \theta \approx \theta$ is valid and Eq. (1) becomes a linear differential equation analogous to the one for the simple harmonic oscillator. In this regime the pendulum oscillates with a period $T_{0}=2 \pi \sqrt{L / g}$, a well-known textbook relation. $\underline{\underline{1}}$ This relation underestimates the exact period for any amplitude, but the difference is almost imperceptible for small angles. For larger angles $T_{0}$ becomes more and more inaccurate for describing the exact period and Eq. (1) can be used to obtain a numerical solution.

Alternatively, an integral expression for the exact pendulum period may be derived from energy considerations, without a detailed discussion of differential equations. If we take the zero of potential energy at the lowest point of the trajectory (see Fig. 1) and choose for simplicity the initial conditions as $\theta(0)=+\theta_{0}$ and $d \theta / d t(0)=0$, we have ${ }^{2}$

$$
m g L\left(1-\cos \theta_{0}\right)=\frac{1}{2} m L^{2}\left(\frac{d \theta}{d t}\right)^{2}+m g L(1-\cos \theta) .
$$

The solution for $d \theta / d t$ is

$$
\frac{d \theta}{d t}= \pm \sqrt{\frac{2 g}{L}\left(\cos \theta-\cos \theta_{0}\right)},
$$

where the $+(-)$ sign is for counter-clockwise (clockwise) motion. Now, integrating $d \theta / d t$ from $\theta_{0}$ to 0 (thus choosing the - sign in Eq. (3)), which corresponds to a time equal to one quarter of the exact period $T$, we have

$$
T=2 \sqrt{2} \sqrt{\frac{L}{g}} \int_{0}^{\theta_{0}} \frac{1}{\sqrt{\cos \theta-\cos \theta_{0}}} d \theta .
$$

The definite integral in Eq. (44) cannot be expressed in terms of elementary functions. $\frac{2.9}{*}$ Note that the numerical evaluation of the period using Eq. (4) is not straightforward because the integrand has a vertical asymptote at $\theta=\theta_{0}$, which makes the integral improper $\stackrel{10}{\underline{10}}$ This difficulty can be circumvented by substituting $\cos \theta$ by $1-2 \sin ^{2}(\theta / 2)$ and making a change of variables given implicitly by $\sin \varphi=\sin (\theta / 2) / \sin \left(\theta_{0} / 2\right)$. In this way, Eq. (4) becomes

$$
T=4 \sqrt{\frac{L}{g}} \int_{0}^{\pi / 2} \frac{1}{\sqrt{1-k^{2} \sin ^{2} \varphi}} d \varphi,
$$


where $k \equiv \sin \left(\theta_{0} / 2\right)$. The definite integral is $K(k)$, the complete elliptic integral of the first kind, which is not improper because $k<1$ for $\left|\theta_{0}\right|<\pi$.

It is not difficult to numerically evaluate $T$ for a given amplitude. The relative error made in approximating $T$ by $T_{0}$, where $T=2 T_{0} / \pi K(k)$, is 11

$$
\frac{T_{0}-T}{T}=\frac{\pi}{2 K(k)}-1
$$

Our proposed approximation for the pendulum period is based on the observation that $f(\varphi, k) \equiv \sqrt{1-k^{2} \sin ^{2} \varphi}$ is a smooth function of $\varphi$, whose concavity changes from downward to upward at a point near the middle of the interval of integration, that is, $0 \leq \varphi \leq$ $\pi / 2$. As shown in Fig. 2, this change occurs for any $\theta_{0}$ between 0 and $\pi / 22^{12}$ We use the points $(0,1)$ and $(\pi / 2, a)$ for a linear interpolation, where $a \equiv f\left(\varphi=\pi / 2, k=\sin \theta_{0} / 2\right)=$ $\sqrt{1-\left(\sin \theta_{0} / 2\right)^{2}}=\cos \theta_{0} / 2$, and approximate $f(\varphi, k)$ by

$$
f\left(\varphi, \theta_{0}\right) \approx 1-\frac{2}{\pi}(1-a) \varphi
$$

We substitute Eq. (17) in the denominator of the integrand in Eq. (5) and find

$$
K(k) \approx \int_{0}^{\pi / 2} \frac{1}{1-(2 / \pi)(1-a) \varphi} d \varphi=-\frac{\pi}{2} \frac{\ln a}{1-a} .
$$

Finally, by substituting Eq. (8) in Eq. (5), we found

$$
T_{\log }=-2 \pi \sqrt{\frac{L}{g}} \frac{\ln a}{1-a}=-T_{0} \frac{\ln a}{1-a} .
$$

Note that $\ln a<0$ and hence $T_{\log }>0$ for $\left|\theta_{0}\right|<\pi$. The relative error in the logarithmic formula in Eq. (9) is given by ${ }^{13}$

$$
\frac{T_{\log }-T}{T}=\frac{\pi}{2 K(k)} \frac{(-\ln a)}{1-a}-1
$$

\section{COMPARISON WITH OTHER APPROXIMATIONS}

We compare the accuracy of our approximation for the pendulum period in Eq. (9) in representing the exact period to that of other known approximations for amplitudes less than or equal to $\pi / 2 .^{12}$ The relative errors found by approximating the exact period by $T_{0}$ and $T_{\log }$, as well as by other formulas, are depicted in Fig. 3, where it is easily seen that all approximations present the same general behavior: For small amplitudes their corresponding 
error curves tend to zero and for larger amplitudes the curves go up monotonically, reflecting the increase of the relative error with the amplitude obtained with all known approximation formulas. However, note that the rate at which the error increases is different for each curve, the smaller rate being the desired one for a good approximation. In this sense, the small angle approximation $T \approx T_{0}$ exhibits the worst behavior because its error becomes greater than $0.1 \%(0.5 \%)$ above an amplitude as low as $7^{\circ}\left(16^{\circ}\right)$.

The second-order approximation found by Bernoulli in 1749 from a perturbative analysis of Eq. (5), perhaps the most famous formula for the large angle period, is 14

$$
T_{2}=T_{0}\left(1+\frac{\theta_{0}^{2}}{16}\right)
$$

As seen from the short-dashed line in Fig. 3, it leads to an error that increases rapidly, overcoming the $0.1 \%(0.5 \%)$ level for amplitudes above $41^{\circ}\left(60^{\circ}\right)$. The addition of more terms improves the accuracy of $T_{2} !^{15}$

More recently, other approximation formulas have been proposed. Among them, the Kidd-Fogg formula has attracted much interest due to its simplicity. ${ }^{8}$ It is given by

$$
T_{K F}=T_{0} \frac{1}{\left.\sqrt{\cos \left(\theta_{0} / 2\right.}\right)} .
$$

The dash-dotted line in Fig. 3 represents the error for $T_{K F}$. The error is greater than $0.1 \%$ only for amplitudes $\theta_{0} \geq 57^{\circ}$ and reaches $0.8 \%$ for $\theta_{0}=90^{\circ}$. Thus, it is not accurate enough for interpreting the experimental data for very large-angle amplitudes, contrary to the claim of Millet. 16

Another formula for the period arises when an interpolation-like linearization is made directly in Eq. (1) 17 The resulting expression is

$$
T_{M}=T_{0}\left(\frac{\sin \theta_{0}}{\theta_{0}}\right)^{-3 / 8},
$$

which has an error greater than $0.1 \%$ only for $\theta_{0} \geq 69^{\circ}$ (see the thin solid curve in Fig. 3). However, the error reaches $\sim 0.4 \%$ for $\theta_{0}=90^{\circ}$, which is four times the typical experimental error $(0.1 \%)$.

The error using Eq. (9) for the period (see the thick solid line in Fig. 3) remains below all other error curves for any $\theta_{0}$ and is greater than $0.1 \%$ only for amplitudes greater than $74^{\circ}$. Moreover, it increases slowly, reaching only $0.2 \%$ for $\theta_{0}=86^{\circ}$. Therefore, our logarithmic formula is the better approximation for the exact pendulum period in the sense it yields the 
smaller relative error and also the smaller rate for the relative error increasing in the range of amplitudes studied here.

\section{EXPERIMENT AND RESULTS}

Reliable data for large-angle pendulum periods were obtained by Fulcher and Davis $\frac{4}{\underline{4}}$ using a pendulum made with piano wire (measuring two successive swings) and by Curtis 18 who determined the period as the average of ten successive periods for each initial amplitude. Both papers are good examples of accurate period measurements made with an ordinary stopwatch. The measurement of the time interval for $n$ successive periods is a good strategy for oscillations in the small angle regime, where the amplitude does not change significantly from a swing to the next, but not for large-angle oscillations, because the period decreases considerably due to air friction. This behavior is confirmed in Fig. 4, where the period $T$ in units of $T_{0}$ is plotted as a function of $\theta_{0}$. In Fig. 4 the curves for each approximation formula discussed in Sec. III are plotted. Experimental data taken from Refs. 4 and 18 and the measurements taken by us in a more sophisticated experiment ${ }^{5.19}$ are also shown. The experimental data for amplitudes greater than $35^{\circ}$ clearly reveal a systematic overestimation for the period due to air damping.

In our experiment both the time-keeping and position detection were done automatically to reduce the instrumental error to milliseconds, which is much less than the error in time keeping when a common stopwatch is used (of the order of $0.2 \mathrm{~s}$, the average human reaction time). We measured the pendulum period by measuring the time interval between two successive passages of the pendulum over the lower point of its circular path, which corresponds to $T / 2$. The measurement was based on the variation of the electrical resistance of a lightdependent resistor during the passing of the pendulum's bob through the path of the light from a laser $\stackrel{\underline{5}}{\underline{5}}$ Electronic circuitry is needed for converting the analogue signal generated in the light-dependent resistor when the pendulum's bob cuts the light's path to TTL compatible digital voltage, so that the microprocessor can understand the change in current. The details of the design/operation of the circuitry and the microprocessor program required for measuring the time interval between successive interruptions in the light-dependent resistor are in Ref. 19.

We devoted much attention to the reduction of the air resistance on the motion of the 
pendulum bob by choosing suitable materials and parameters for the pendulum. We used lead as the bob material due to its high density in comparison to other inexpensive metals, which gives a small size and large weight for the bob. We found that a cylinder is preferable to a sphere because it allows for a better localization of the center of mass, which is needed for measuring $L$ accurately. The cylindrical shape also yields a reduction of air resistance by reducing the scattering cross-section, that is, by choosing a diameter much smaller than the height of the cylinder. These considerations led us to fabricate a body with a mass of $0.400 \mathrm{~kg}$. For this massive bob we verified that cords made of nylon, a commonly used material, are inadequate because they stretch considerably for large angle oscillations and cause undesirable vibrations. The more convenient material taking into account low elasticity, lightness (see Ref. 20 for the importance of this factor), price, and availability, seems to be cotton. We used a common sewing thread as the pendulum cord. We also investigated what cord length would give the best experimental results for large angles. ${ }^{21}$ After comparing many lengths for an amplitude of $60^{\circ}$, we choose a length of $1.50 \mathrm{~m}$ so that the bob speed would be small (because air friction increases with speed, the longer the string length, the less the effect of air friction on the period) This length has a period of $\approx 2.5 \mathrm{~s}$, which is sufficiently small for doing several repetitions of the period measurement for each amplitude during a typical one-hour class. These considerations led us to much more accurate experimental data for the pendulum period for amplitudes less than or equal to $90^{\circ}$ as shown in Fig. 4. It is seen that our experimental data (black diamonds) are closer to the exact period expected in the absence of air resistance (the solid line) than the data in Refs. 4 (crosses) and 18 (circles). The logarithmic formula in Eq. (91) is also in better agreement with the experimental data.

\section{Acknowledgments}

F.M.S.L. thanks Prof. Getúlio T. Brasil (UniCEUB, Brasília-DF) for assistance on the experimental setup.

* Electronic address: fabio@fis.unb.br 
1 R. A. Serway and R. J. Beichner, Physics for Scientists and Engineers (Harcourt Brace, Orlando, FL, 2000), 5th ed., pp. 402-404.

2 S. T. Thornton and J. B. Marion, Classical Dynamics of Particles and Systems (Brooks/Cole, New York, 2004), 5th ed., pp. 155-158. See also Appendix B for a good presentation of elliptic integrals.

3 It seems the only exception is the pendulum of antique astronomical clocks, whose amplitude is less than $1.5^{\circ}$, as pointed out in A. Sommerfeld, Mechanics (Academic Press, New York, 1952), p. 90 .

4 L. P. Fulcher and B. F. Davis, "Theoretical and experimental study of the motion of the simple pendulum," Am. J. Phys. 44, 51-55 (1976).

5 N. Aggarwal, N. Verma, and P. Arun, "Simple pendulum revisited," Eur. J. Phys. 26, 517-523 (2005).

6 T. Araki, "Measurement of simple pendulum motion using flux-gate magnetometer," Am. J. Phys. 62, 569-571 (1994).

7 P. Moreland, "Improving precision and accuracy in the $g$ lab," Phys. Teach. 38, 367-369 (2000).

8 R. B. Kidd and S. L. Fogg, "A simple formula for the large-angle pendulum period," Phys. Teach. 40, 81-83 (2002).

9 M. Abramowitz and I. A Stegun, Handbook of Mathematical Functions (Dover, New York, 1968), p. 589 .

10 S. D. Schery, "Design of an inexpensive pendulum for study of large-angle motion," Am. J. Phys. 44, 666-670 (1976).

11 This error depends on $\theta_{0}$ implicitly (through $k$ ) and its absolute value increases rapidly with it. For instance, $T_{0}$ underestimates the exact period with an error of $15.3 \%$ for an amplitude of $\pi / 2 \mathrm{rad}$.

12 Of course, the cases with $\theta_{0}>\pi / 2$ rad are of less interest because most simple pendulum experiments in introductory physics labs are done with flexible cords instead of rigid rods, which prevents the pendulum bob from following a circular path soon after it is released. However, our approximate formula is more accurate than other ones even for $\theta_{0}>\pi / 2 \mathrm{rad}$.

13 Note that the error with respect to the exact period $T$, for each amplitude, is, naturally, the quantity to be analyzed here instead of the error with respect to $T_{0}$.

14 C. J. Smith, "A Degree Physics, (Part I; The General Properties of Matter)", Edward Arnold 
Ltd, London 1960.

15 R. R. Parwani, "An approximate expression for the large-angle period of a simple pendulum," Eur. J. Phys. 25, 37-39 (2004).

16 L. E. Millet, "The large-angle pendulum period," Phys. Teach. 41, 162-163 (2003).

17 M. I. Molina, "Simple linearization of the simple pendulum for any amplitude," Phys. Teach. 35, 489-490 (1997).

18 R. K. Curtis, "The simple pendulum experiment," Phys. Teach. 19, 36 (1981).

19 For a version of Ref. 5 that is richer in experimental details, see pre-print phys-0409086 (2004), available at xxx.lanl.gov.

20 H. L. Armstrong, "Effect of the mass of the cord on the period of a simple pendulum," Am. J. Phys. 44, 564-566 (1976). See also the comment in S. T. Epstein and M. G. Olsson, Am. J. Phys. 45, 671-672 (1977).

21 The lengths were measured after tying up the thread firmly to a hook in the ceiling lab, at one end, and to a small ring at the top of the lead cylinder, at the other end.

22 T. H. Fay, "The pendulum equation," Int. J. Math. Educ. Sci. Technol. 33, 505-519 (2002).

23 S. C. Zilio, "Measurement and analysis of large-angle pendulum motion," Am. J. Phys. 50, 450-452 (1982). 


\section{Figure Captions}

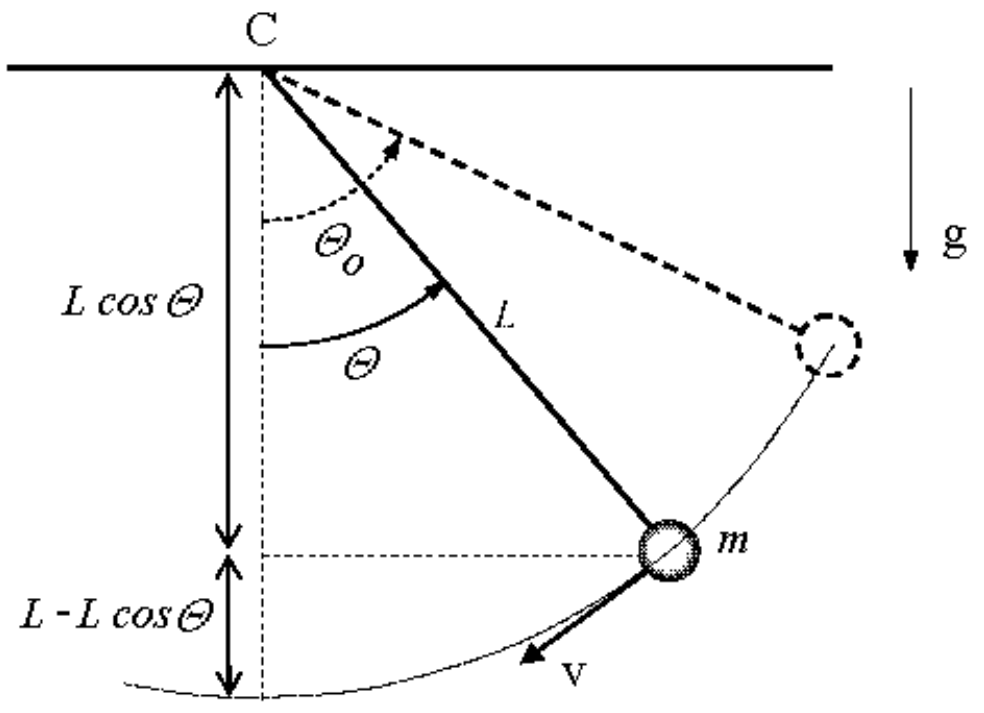

FIG. 1: The pendulum bob is released at rest from a position that forms an angle $\theta_{0}$ with the vertical and passes at an arbitrary angle $\theta\left(<\theta_{0}\right)$ with a velocity $L d \theta / d t$. Its height depends on $\theta$ according to $L(1-\cos \theta)$. 


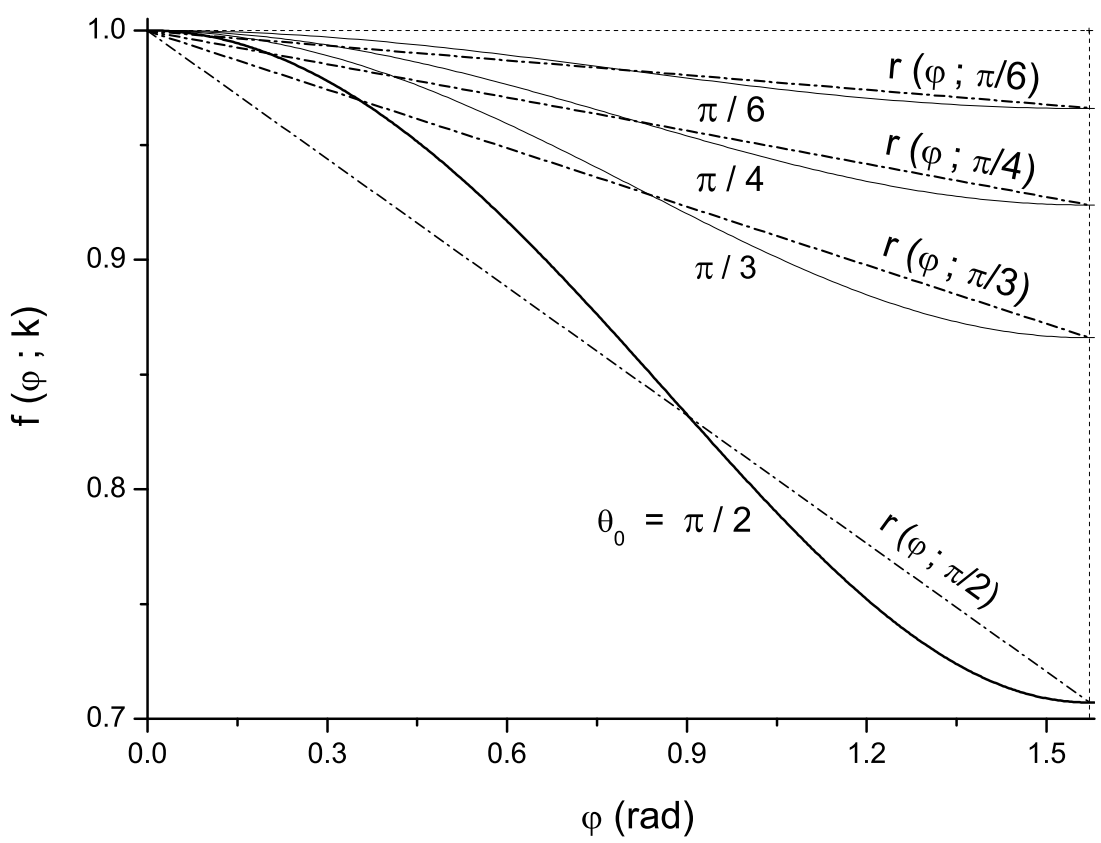

FIG. 2: Behavior of the function $f(\varphi, k)=\sqrt{1-k^{2} \sin ^{2} \varphi}$ for $0 \leq \varphi \leq \pi / 2 \mathrm{rad}$ and for some values of $\theta_{0}\left(k=\sin \left(\theta_{0} / 2\right)\right)$. The horizontal and vertical dashed lines are for $f(\varphi, k)=1$ and $\varphi=\pi / 2$ rad, respectively. The dash-dotted lines are the linear interpolation in Eq. (7) for $\theta_{0}=\pi / 6, \pi / 4$, $\pi / 3$, and $\pi / 2 \mathrm{rad}$. 


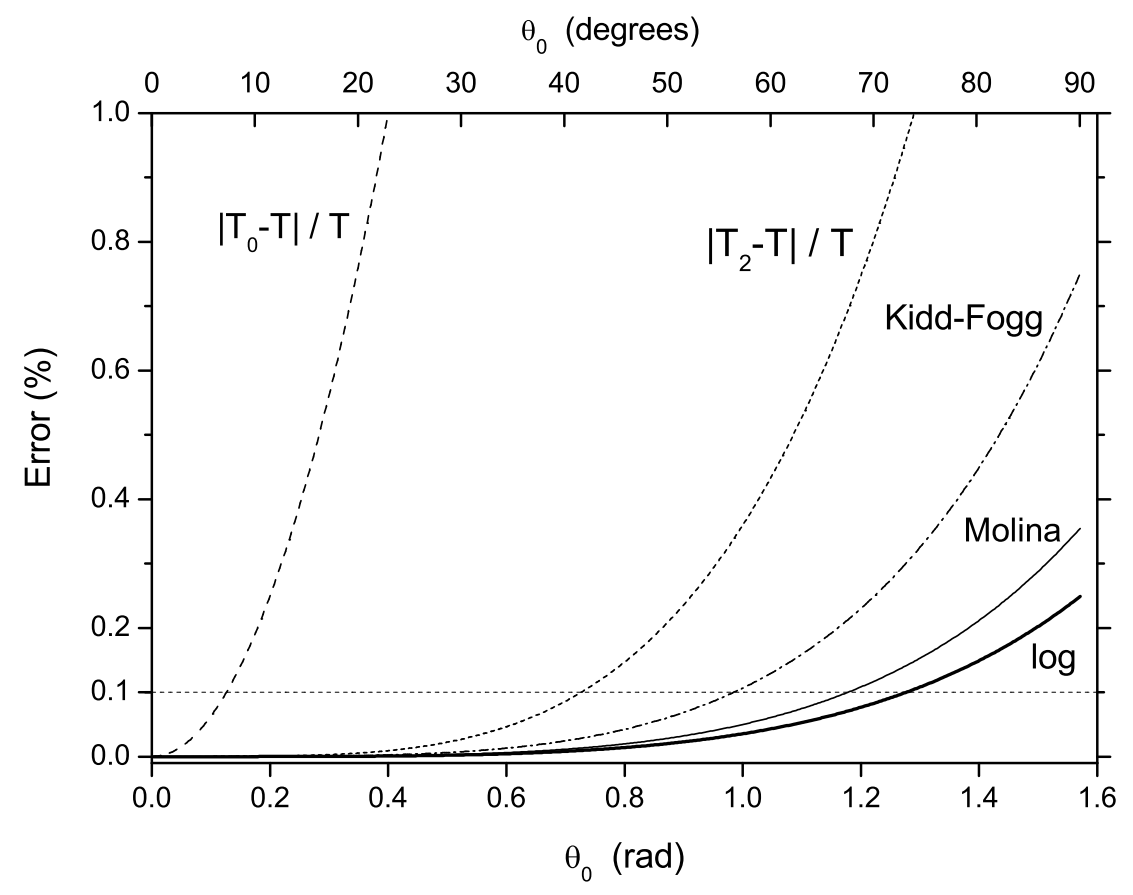

FIG. 3: Comparison of the relative errors for the various approximations discussed in the text for the period. All curves increase monotonically with $\theta_{0}$. The horizontal dashed line marks the $0.1 \%$ level. The small angle approximation $\left(T \approx T_{0}\right)$ yields an error that is greater than $0.1 \%$ for $\theta_{0}>7^{\circ}$ and reaches $15.3 \%$ for $\theta_{0}>90^{\circ}$. The thick solid line is for Eq. (9). Note that it remains below all other curves for $0^{\circ} \leq \theta_{0} \leq 90^{\circ}$. 


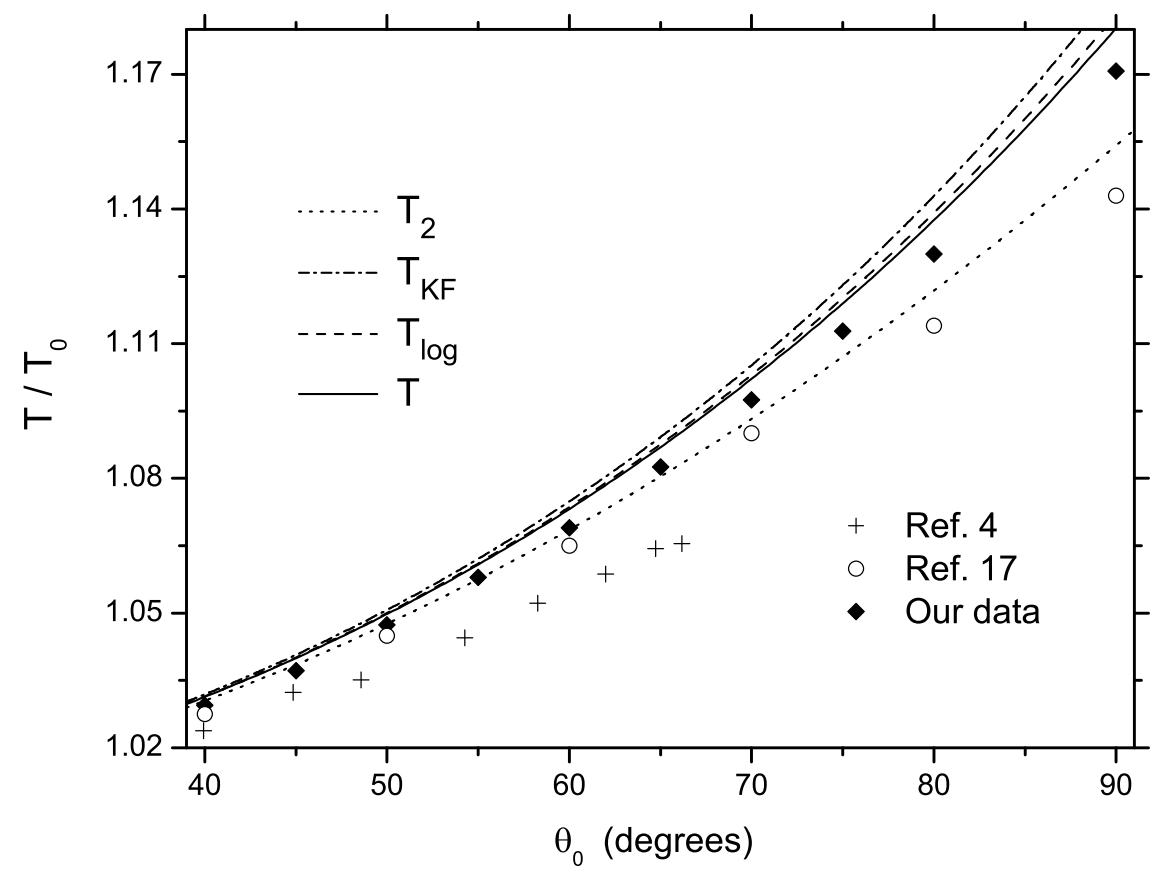

FIG. 4: Comparison of the ratio $T / T_{0}$ for the approximation formulas discussed in the text and experimental data. The dotted curve is for the Bernoulli formula, Eq. (11). The dash-dotted curve is for the Kidd-Fogg formula, Eq. (12). The dashed line is for our logarithmic formula, Eq. (9). The solid line is the curve for the exact period, found by numerical integration of $K(k)$. The experimental data were taken from Ref. $4(+)$ and Ref. 18 (०), and the black diamonds are our experimental data. 